\title{
2D NMR-based Metabolomics with HSQC/TOCSY NOAH \\ Supersequences
}

\begin{abstract}
Alexandar L. Hansen, ${ }^{1}$ Ëriks Kupče, ${ }^{2}$ Da-Wei Li, ${ }^{1}$ Lei Bruschweiler-Li, ${ }^{1}$ Cheng Wang ${ }^{3}$ Rafael Brüschweiler ${ }^{1,3,4}$

${ }^{1}$ Campus Chemical Instrument Center, ${ }^{2}$ Bruker UK Ltd., Banner Lane, Coventry, CV4 9GH, U.K. ${ }^{3}$ Department of Chemistry and Biochemistry, ${ }^{4}$ Department of Biological Chemistry and Pharmacology, The Ohio State University, Columbus, Ohio 43210, U.S.A.
\end{abstract}

\section{Contact information}

Alexandar L Hansen, E-mail: hansen.434@osu.edu

"To whom correspondence should be addressed

\section{Table of Contents}

The zz-filter is an isotope-selective composite pulse ........................................... 2

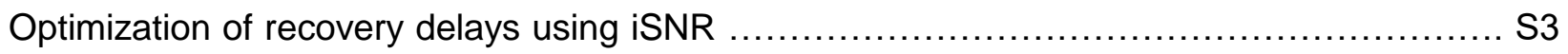

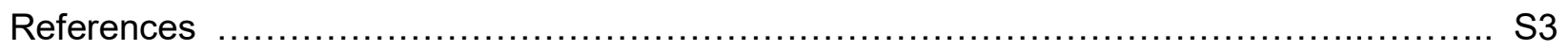

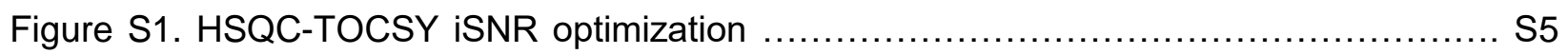

Figure S2. Peak Volume comparison of NOAH2 TOCSYs ..................................... 6

Figure S3. Peak Volume Comparison of NOAH3 TOCSYs ..................................... 6

Figure S4. Peak Volume comparison of NOAH HSQCs …................................. S7

Figure S5. Peak Volume Comparison of NOAH HSQC-TOCSYs ............................ S7

Figure S6. Un-overlaid version of Figure 3. ............................................... S8

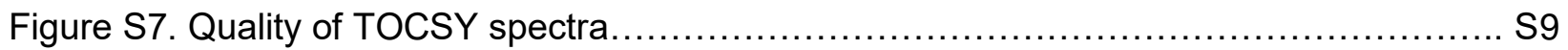


The pulse sequences are available from Dr. Alexandar Hansen upon request and will also be deposited in the Bruker User Library.

\section{The zz-filter is an isotope-selective composite pulse}

We first illustrate the problem when combining a sensitivity-enhanced HSQC with 'unlabeled' proton preservation. To do this, it is easiest to work backwards from the acquisition and analyze the effect of the reverse transfer scheme on these protons (Fig 1B of the main text, point $a$ to $b$ ). It can be easily shown that transverse 'unlabeled' proton magnetization will remain transverse following the magnetization transfer, whereas z magnetization will likewise be left unchanged.

$$
\begin{aligned}
& H_{x} \stackrel{90 x-\delta-180 x-\delta-90 y}{\longrightarrow}-H_{z} \stackrel{\Delta-180 x-\Delta-90 x}{\longrightarrow}-H_{y} \\
& H_{y} \stackrel{90 x-\delta-180 x-\delta-90 y}{\longrightarrow}-H_{x} \stackrel{\Delta-180 x-\Delta-90 x}{\longrightarrow}-H_{x} \\
& H_{z} \stackrel{90 x-\delta-180 x-\delta-90 y}{\longrightarrow} H_{y} \stackrel{\Delta-180 x-\Delta-90 x}{\longrightarrow}-H_{z}
\end{aligned}
$$

This differs from the unenhanced HSQC where acquisition occurs following the first transfer period when 'unlabeled' proton magnetization can be placed along z, shown in row the first row. Since the preservation of equivalent pathways used for sensitivity enhancement ${ }^{1}$ dictates the phases of pulses used in the transfer, the goal is to ensure that 'unlabeled' proton magnetization is already along $z$ before this transfer begins. This is often achieved in NMR using selective pulses, for instance when preservation of the water signal is desired. However, because there is no frequency difference between 'labeled' and 'unlabeled' protons, a frequency-selective pulse does not work here. Instead, it requires an 'isotope-selective' pulse.

To achieve isotope selection a composite-pulse element (Fig 1B, box), which incorporates an adjustable pulse phase $\Psi$ at the end of a $z z-$ filter $^{2}$, is used to replace the initial $90^{\circ}{ }^{1} \mathrm{H}$ pulse of the sensitivity-enhanced HSQC sequence. At first glance it appears like a double-BIRD filter element. ${ }^{3,4}$ However, instead of echo lengths of $1 / \mathrm{J}_{\mathrm{CH}}$, the echoes are of length $1 /\left(2 \mathrm{~J}_{\mathrm{CH}}\right)$ equivalent to a pair of INEPT sequences. We refer to this $z z$ isotope-selective pulse element as ZIP. To highlight the difference ZIP makes for the 'unlabeled' magnetization, it is useful to follow the magnetization of protons directly bound to either a ${ }^{12} \mathrm{C}$ or ${ }^{13} \mathrm{C}$ nucleus. In the former ('unlabeled') case, the application of carbon pulses is irrelevant, so the magnetization flows as follows before the final pulse:

$$
H_{z, U} \stackrel{90^{\circ} H_{x}-\tau-180^{\circ} H_{x}-\tau-90^{\circ} H_{x}}{\longrightarrow} H_{z, U} \stackrel{\tau-180^{\circ} H_{x}-\tau}{\longrightarrow}-H_{z, U}
$$


The net effect is merely an inversion of ${ }^{12} \mathrm{C}$-bound protons. By contrast, the evolution of the 'labeled', ${ }^{13} \mathrm{C}$-bound protons proceed as:

$$
H_{z, L} \stackrel{90^{\circ} H_{x}}{\longrightarrow}-H_{y, L} \stackrel{\tau-180^{\circ} H_{x} / C_{x}-\tau}{\longrightarrow}-2 H_{x, L} C_{z} \stackrel{90^{\circ} H_{x}}{\longrightarrow}-2 H_{x, L} C_{z} \stackrel{\tau-180^{\circ} H_{x} / C_{x}-\tau}{\longrightarrow} H_{y, L}
$$

Here, the effect is an isotope-selective $90^{\circ}-x$ pulse. It is worth noting that the ZIP sequence is a point-to-point optimization and, while reversible, does not work as a $90^{\circ}-x$ pulse for other initial magnetizations. By selecting $\psi$ to be of phase $-y$ at the end of the ZIP sequence, the resulting 'labeled' proton magnetization is unchanged, while 'unlabeled' protons are placed along - $x$. Since the final ${ }^{1} \mathrm{H}$ pulse of the INEPT has also y phase, the 'unlabeled' protons simply experience a short echo and return to $+z$ after the $t_{1}$ period, while the 'labeled' proton magnetization is transferred in the usual manner to anti-phase carbon magnetization. Following chemical shift encoding, the sensitivity-enhanced reverse transfer scheme also has a net-zero effect on the 'unlabeled' proton magnetization, as illustrated above, so that it is brought once again along $+z$ before acquisition. Extending this interpretation to the $z z-H M B C$ sequence, ${ }^{2}$ one just recognizes that the choice of $\psi$ in the zz-filter is $x$ phase and has the desired effect of placing 'labeled' proton magnetization along $+z$ while 'unlabeled' protons experience a $90^{\circ}-x$ pulse.

\section{Optimization of recovery delays using iSNR}

Recovery delays for use with ASAP acquisition was accomplished with short 2D experiments ( 10-30min) that were recorded with varying $\mathrm{d} 1$ and $\mathrm{NS}$ (8 or 16 scans, depending on $\mathrm{d} 1$ ). These experiments were processed and then 1D projections were generated and baselinecorrected, as projections tend to have an offset of the noise level. These projections were integrated according to region (aromatic, aliphatic) using a modified Bruker macro, tabulated and converted to an iSNR value using the number of scans and the exact experiment time. Since entire spectral regions were analyzed in this way, rather than only few selected peaks, the results are expected to be sufficiently general.

\section{References}

(1) Rance, M. Sensitivity Improvement in Multidimensional NMR Spectroscopy. Bull. Magn. Reson. 1994, 16 (1-2), 54-67.

(2) Kupče, E.; Claridge, T. D. W. New NOAH Modules for Structure Elucidation at Natural Isotopic Abundance. J. Magn. Reson. 2019, 307, 106568. 
https://doi.org/10.1016/j.jmr.2019.106568.

(3) Garbow, J. R.; Weitekamp, D. P.; Pines, A. Bilinear Rotation Decoupling of Homonuclear Scalar Interactions. Chem. Phys. Lett. 1982, 93 (5), 504-509. https://doi.org/10.1016/0009-2614(82)83229-6.

(4) Breeze, A. L. Isotope-Filtered NMR Methods for the Study of Biomolecular Structure and Interactions. Prog. Nucl. Magn. Reson. Spectrosc. 2000, 36 (4), 323-372. https://doi.org/10.1016/S0079-6565(00)00020-0. 


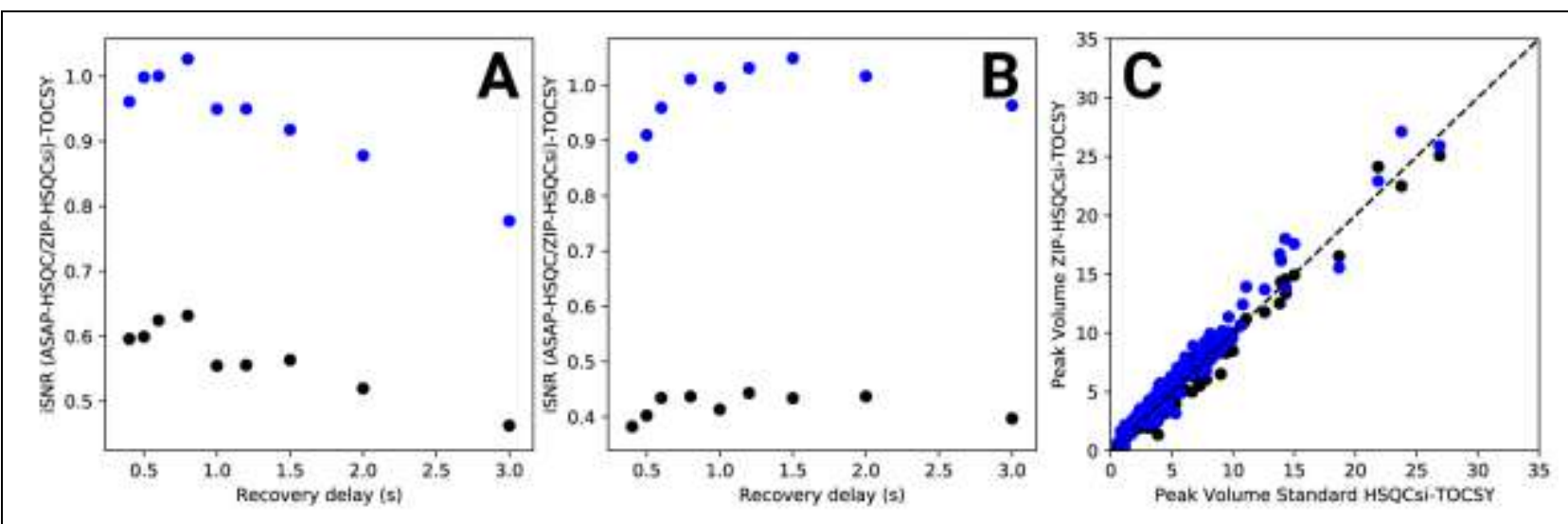

Figure S1. HSQC-TOCSY iSNR optimization of the recovery delay from projections of aliphatic $(A)$ and aromatic $(B)$ regions and scaled relative to the standard Bruker sequence recorded with a $1.5 \mathrm{~s}$ recovery delay. The ASAP-HSQC-TOCSY sequence by Luy is shown in black, while the ZIP-HSQC-TOCSY is shown in blue. (C) Peak volumes for individual peaks (y-axis) compared to those of the standard Bruker sequence (x-axis) recorded with a $1.5 \mathrm{~s}$ recovery delay and 8 scans for a total experiment time of $245 \mathrm{~min}$. In black, the ZIP-HSQC-TOCSY was recorded with $1.5 \mathrm{~s}$ recovery delay and 8 scans for a total of $247 \mathrm{~min}$, while in blue the experiment was recorded with 0.75 s recovery and 12 scans for a total of $216 \mathrm{~min}$. 

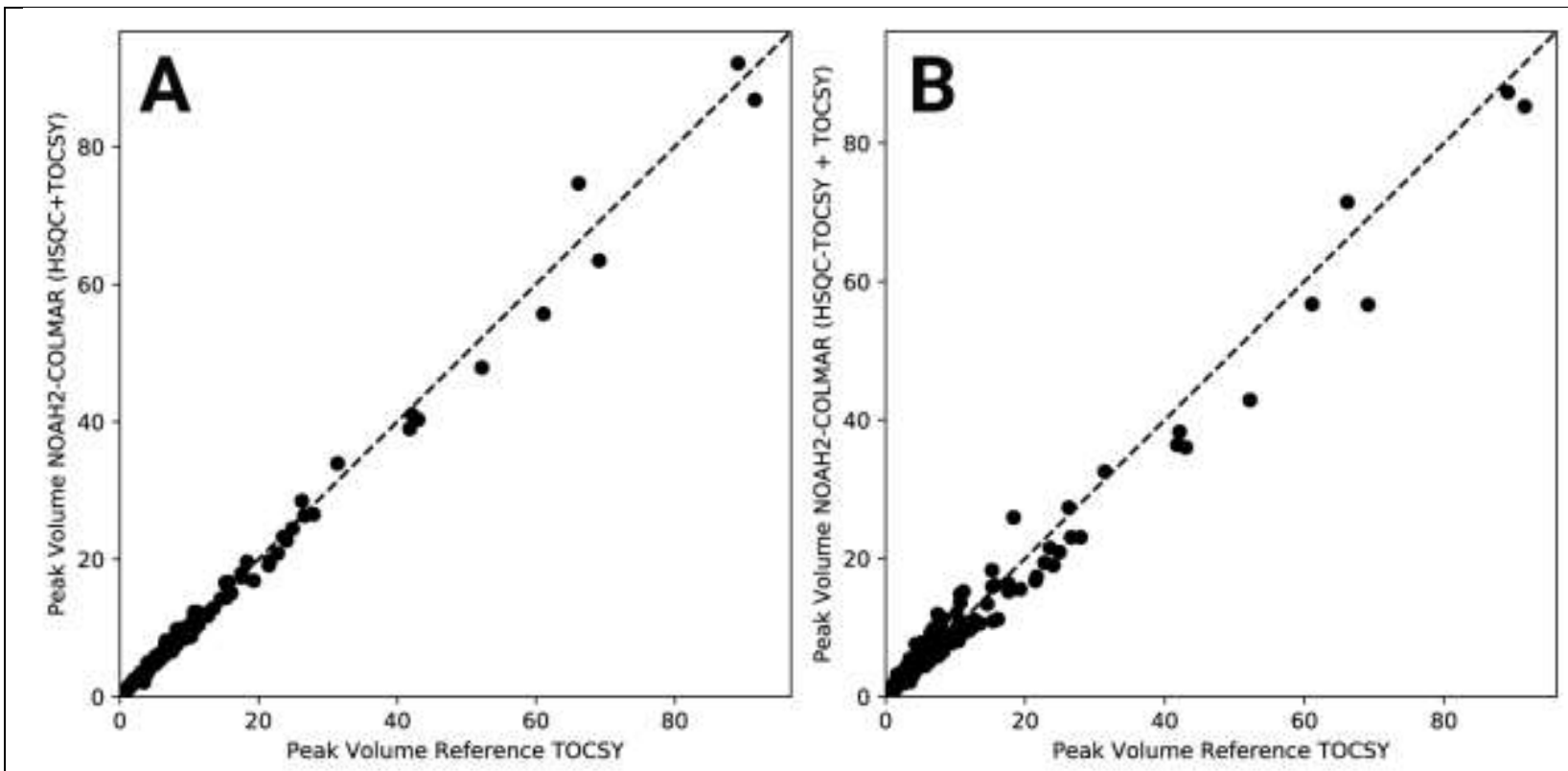

Figure S2. Peak volumes of the TOCSY component of the NOAH2-COLMAR HSQC + TOCSY (A) and HSQC-TOCSY + TOCSY (B) experiments compared to the reference Bruker sequence. The NOAH2 experiments were recorded for $275 \mathrm{~min}(\mathrm{~A})$ and $302 \mathrm{~min}(\mathrm{~B})$.
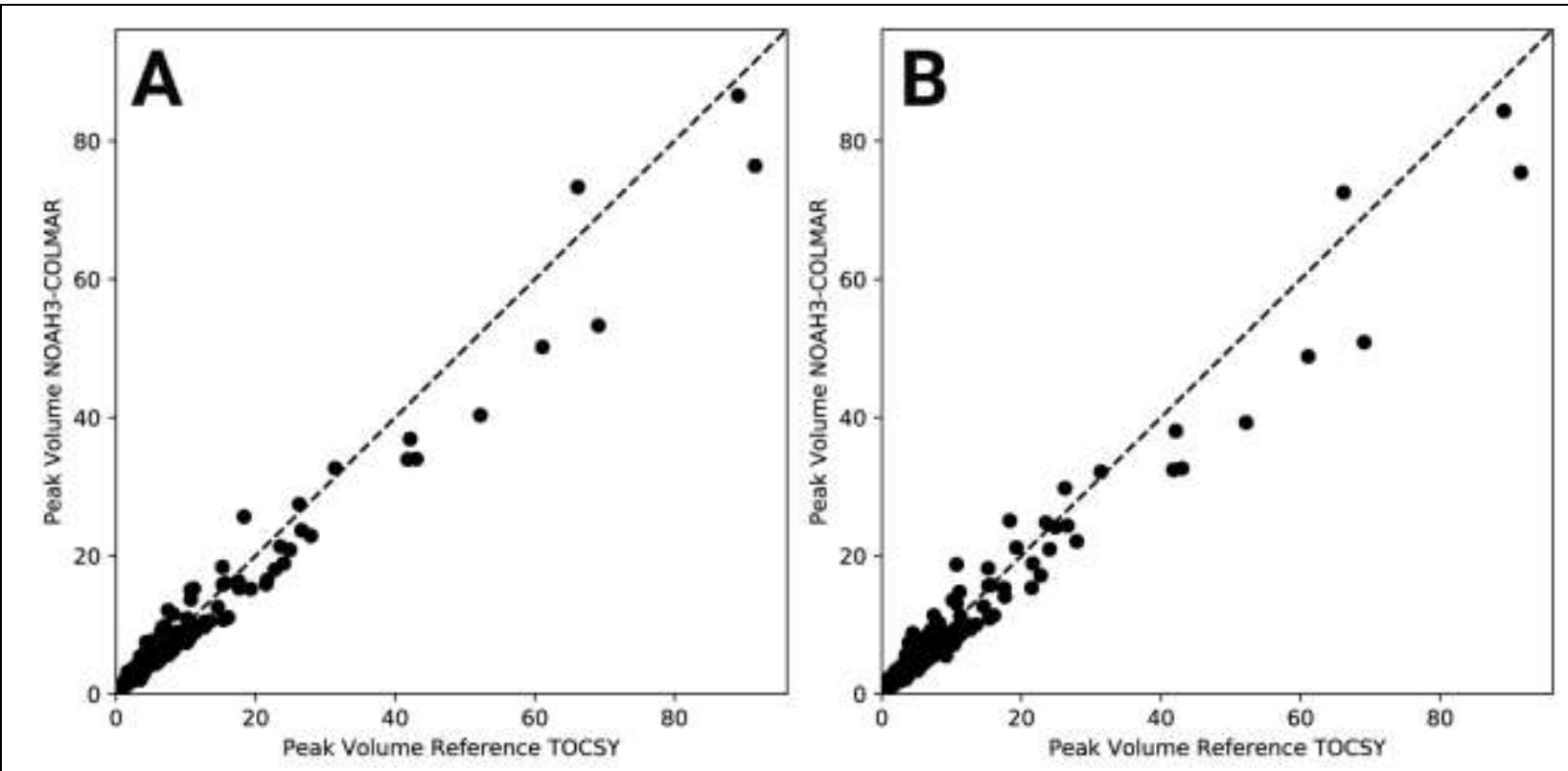

Figure S3. Peak volumes of the TOCSY component of the NOAH3-COLMAR HSQC-TOCSY + HSQC + TOCSY (A) and HSQC + HSQC-TOCSY + TOCSY (B) experiments compared to the reference Bruker sequence. The NOAH3 experiments were recorded in 328 and 336 minutes in $A$ and $B$, respectively. 

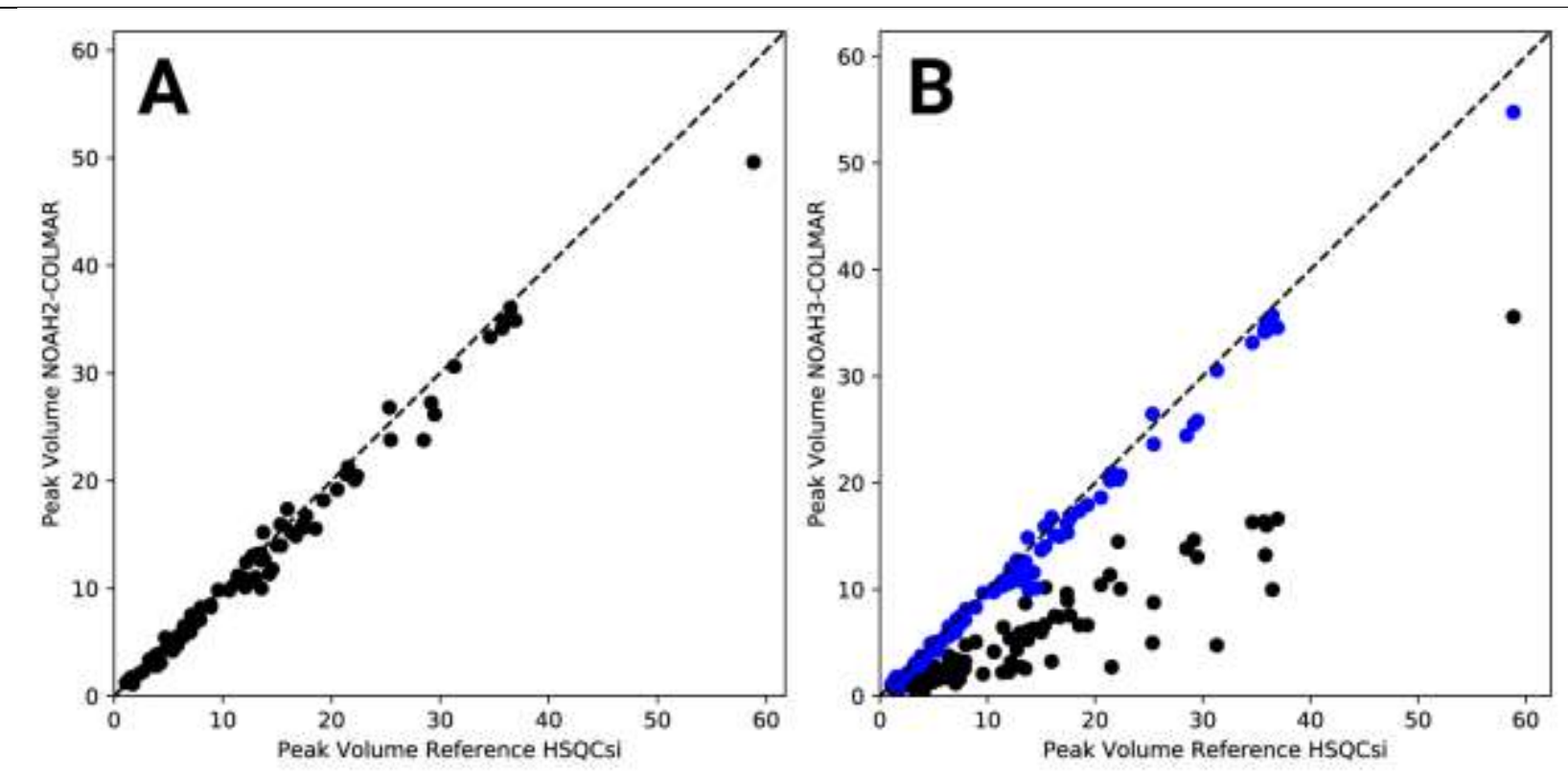

Figure S4. Peak volumes of the HSQC component of the NOAH2-COLMAR (A) and NOAH3-COLMAR (B) HSQC-TOCSY + HSQC + TOCSY (black) and HSQC + HSQC-TOCSY + TOCSY (blue) experiments compared to the reference Bruker sequence. The NOAH2 experiment was recorded in $275 \mathrm{~min}$. The NOAH3 experiments were recording in 328 and 336 minutes in black and blue, respectively.
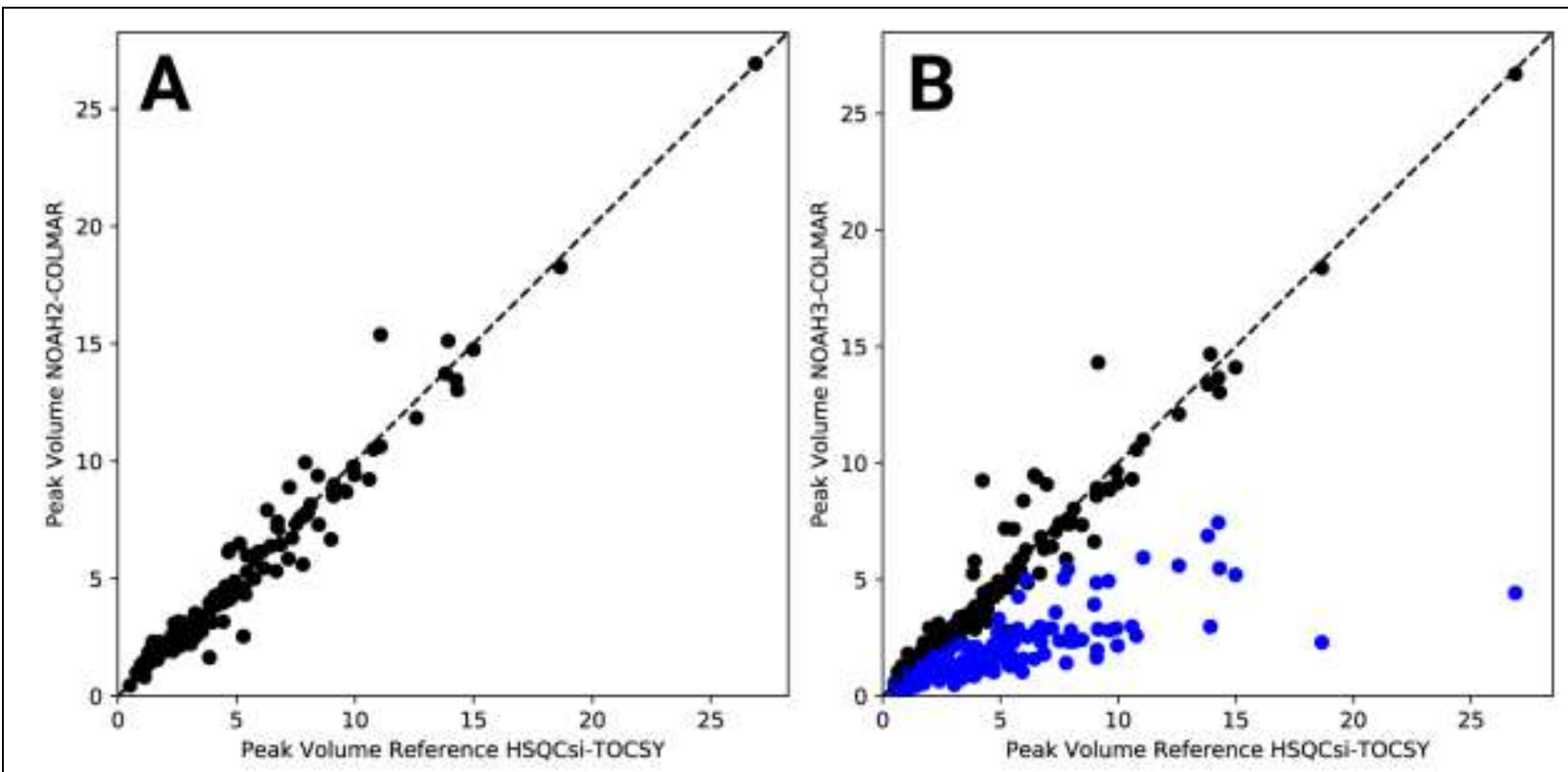

Figure S5. Peak volumes of the HSQC-TOCSY component of the NOAH2-COLMAR (A) and NOAH3COLMAR (B) HSQC-TOCSY + HSQC + TOCSY (black) and HSQC + HSQC-TOCSY + TOCSY (blue) experiments compared to reference sequential experiments. The NOAH2 experiment was recorded in 302 minutes. The NOAH3 experiments were recorded in 328 and 336 minutes in black and blue, respectively. 

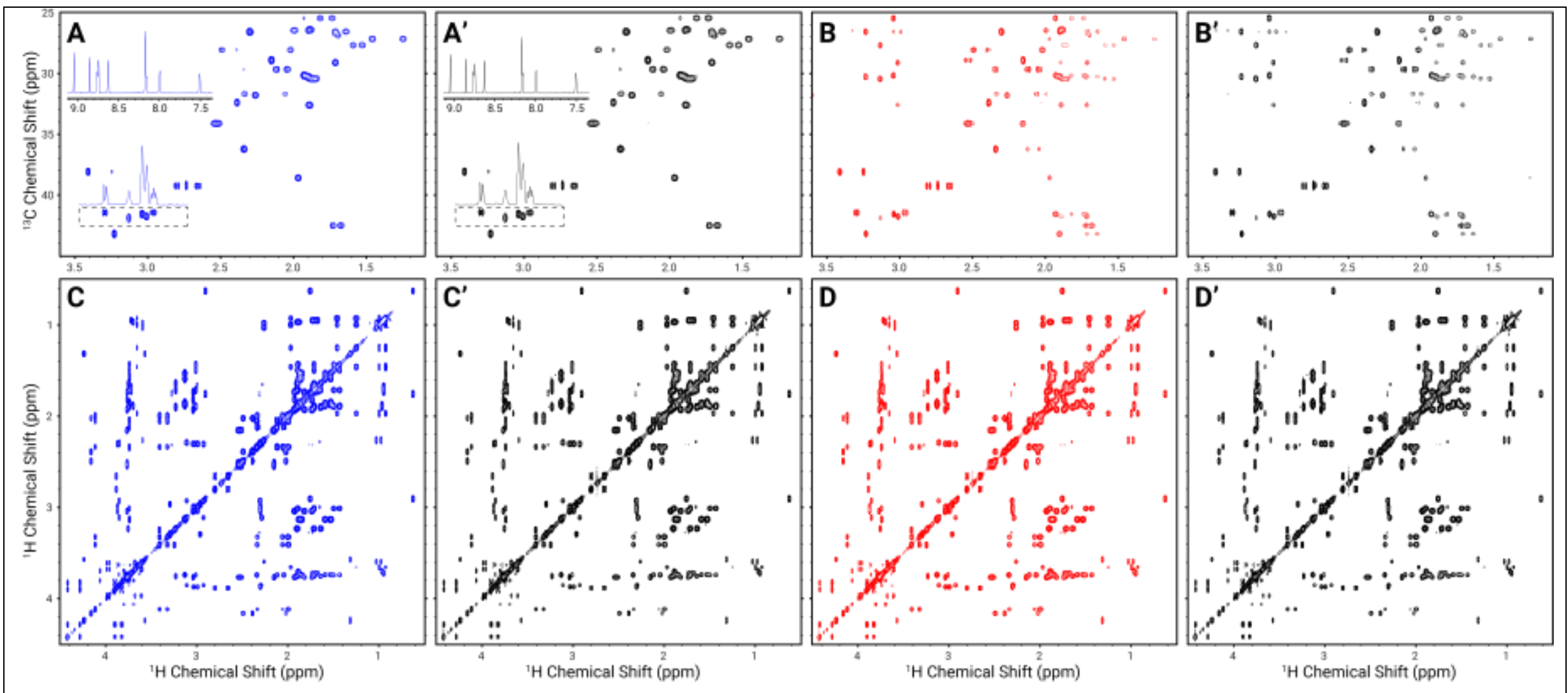

${ }^{1} \mathrm{H}$ Chemical Shift (ppm)
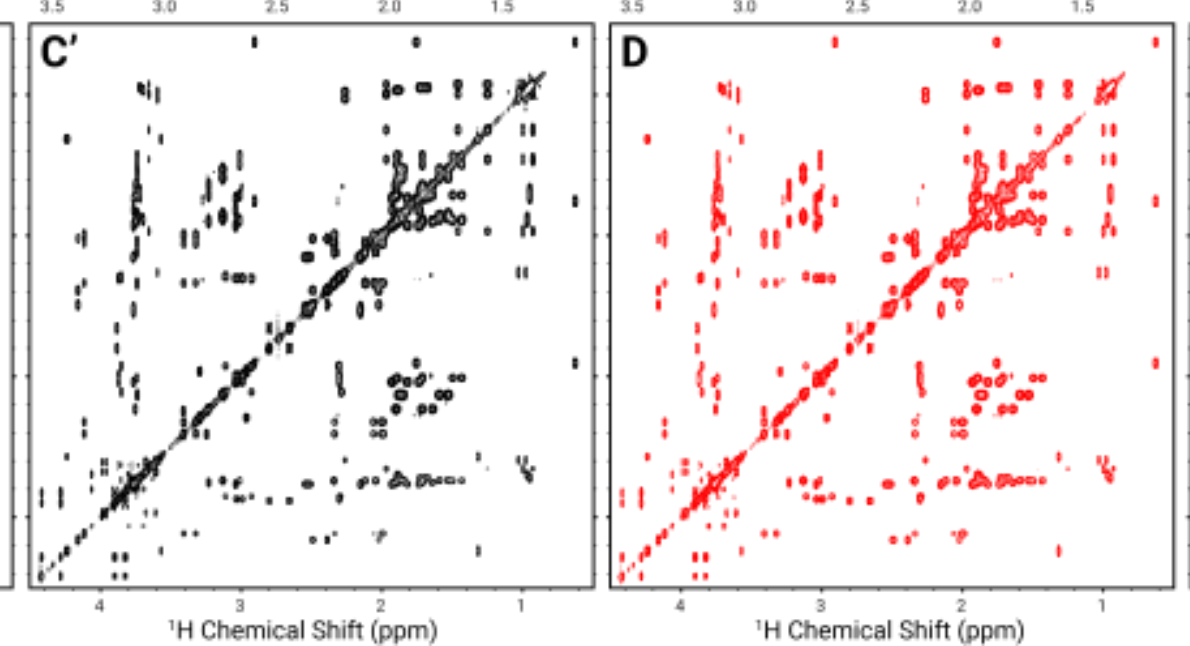

${ }^{1} \mathrm{H}$ Chemical Shift (ppm)

Figure S6. Un-overlaid version of Figure 3. In color (A, B, C, D) are the spectra from the NOAH sequences as described in Figure 3. In black (A', B', C', D') are the spectra from the standard sequences. Figures C' and D' are the same TOCSY spectrum. 


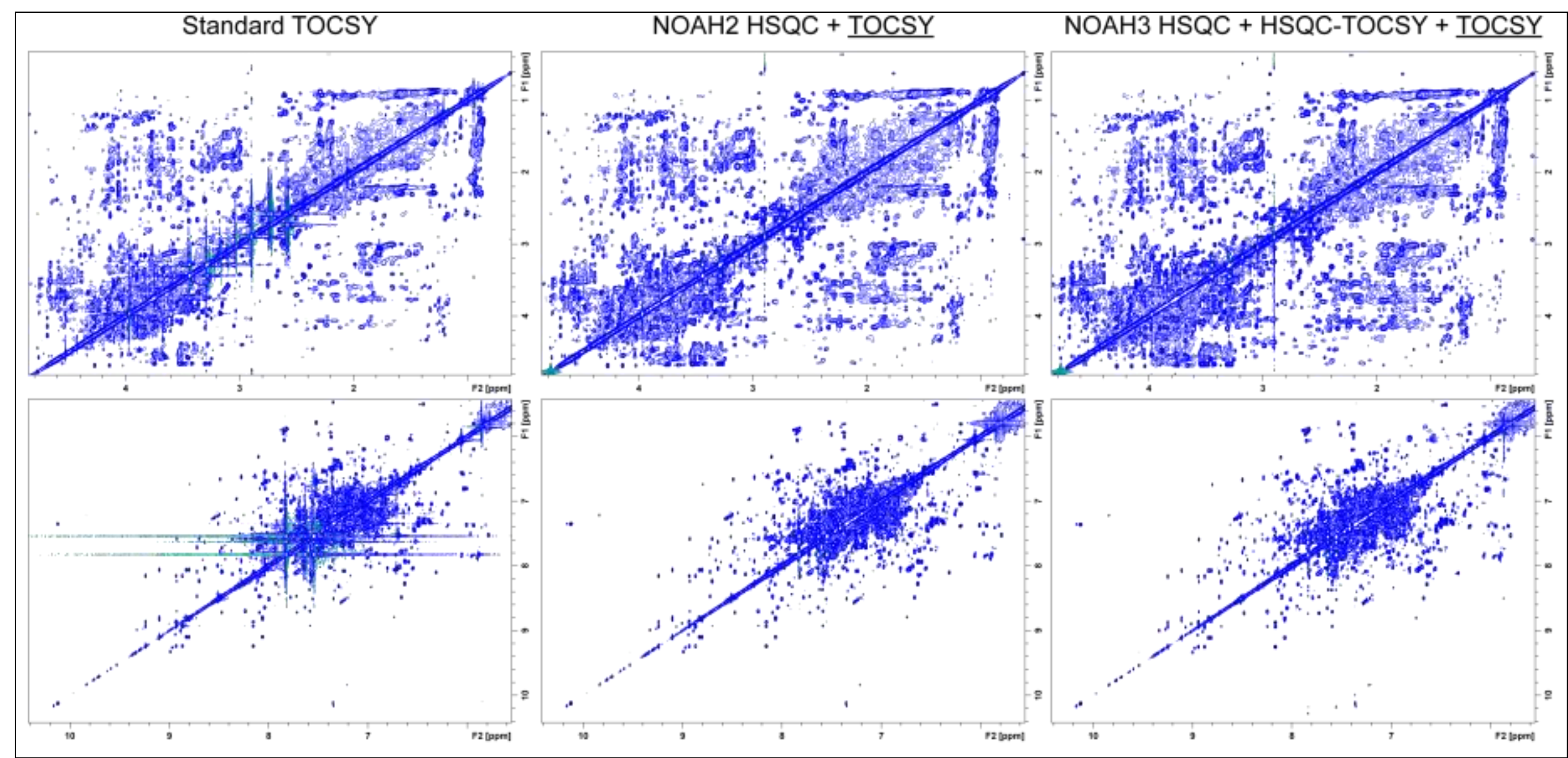

Figure S7. TOCSY spectra recorded for mouse urine using the standard and NOAH sequences, as is indicated above the plots. The top row shows the aliphatic region while the bottom shows the aromatic region. NOAH has noticeably fewer artifacts, such as lower $\mathrm{t}_{1}$-noise and phasing artifacts, compared to the standard sequence. 